\title{
Compensation of the Skin Effect in Low-Frequency Potential Drop Measurements
}

\author{
J. $\operatorname{Corcoran}^{1}$ (1) P. B. Nagy ${ }^{1,2}$
}

Received: 3 May 2016 / Accepted: 22 September 2016 / Published online: 6 October 2016

(C) The Author(s) 2016. This article is published with open access at Springerlink.com

\begin{abstract}
Potential drop measurements are routinely used in the non-destructive evaluation of component integrity. Potential drop measurements use either direct current (DC) or alternating current $(\mathrm{AC})$, the latter will have superior noise performance due to the ability to perform phase sensitive detection and the reduction of flicker noise. AC measurements are however subject to the skin effect where the current is electromagnetically constricted to the surface of the component. Unfortunately, the skin effect is a function of magnetic permeability, which in ferromagnetic materials is sensitive to a number of parameters including stress and temperature, and consequently in-situ impedance measurements are likely to be unstable. It has been proposed that quasi-DC measurements, which benefit from superior noise performance, but also tend to the skin-effect independent DC measurement, be adopted for in-situ creep measurements for power station components. Unfortunately, the quasi-DC measurement will only tend to the DC distribution and therefore some remnant sensitivity to the skin effect will remain. This paper will present a correction for situations where the remnant sensitivity to the skin effect is not adequately suppressed by using sufficiently low frequency; the application of particular interest being the in-situ monitoring of the creep strain of power station components. The correction uses the measured phase angle to approximate the influence of the skin effect and allow recovery of the DC-asymptotic value of the resistance. The basis of the correction, that poten-
\end{abstract}

$凶$ J. Corcoran

joseph.corcoran07@imperial.ac.uk

1 Department of Mechanical Engineering, Imperial College London, London SW7 2AZ, UK

2 Department of Aerospace Engineering and Engineering Mechanics, University of Cincinnati, Cincinnati, OH 45221-0070, USA tial drop measurements are minimum phase is presented and illustrated on two cases; the creep strain sensor of practical interest and a conducting rod as another common case to illustrate generality. The correction is demonstrated experimentally on a component where the skin effect is manipulated by application of a range of elastic stresses.

Keywords Potential drop - ACPD - DCPD - Skin effect . Creep monitoring $\cdot$ Minimum phase

\section{Introduction}

Potential drop (PD) measurements have been in frequent use for non-destructive evaluation (NDE) inspection $[1,2]$ and for fundamental material studies [3-5] for decades. Four electrodes are connected to the surface of a conducting component, current is injected through two of the electrodes while the potential difference is monitored across the remaining two electrodes, the electrical transfer impedance is then calculated. Usually resistance, the real part of impedance, is used to infer changes in geometry arising from component strain $[6,7]$ or defect growth [8-10], or less frequently changes in electrical conductivity $[11,12]$.

Potential drop measurements are broadly categorised depending on the use of alternating or direct current (AC and DC respectively), the choice of which depends on the utilisation of the skin-effect. In AC measurements the current is electromagnetically constricted to the surface of the component, restricting the region that is interrogated. The skin effect results in an exponentially decreasing current density with depth. The electromagnetic skin-depth, $\delta$, the depth at which the current density is $1 / e(\sim 37 \%)$ of its surface density, is given by the equation, 
$\delta=\frac{1}{\sqrt{\sigma \pi f \mu}}$,

where $\sigma$ is the electrical conduictivity, $f$ is the current frequency and $\mu$ is the magnetic permeability of the component.

The NDE application of particular interest is the potential drop creep strain sensor for use in high-temperature, highpressure, static power station components $[6,7,13]$. A square configuration of electrodes is permanently attached to the surface of a component. As the electrodes move together with the surface, when the component strains the changing distances between the electrodes will alter the measured resistance. In ferromagnetic materials, which are very common across a wide range of engineering applications, including power station pipework, the magnetic permeability is a function of many different parameters including temperature, stress, thermal history, cold work and alloy composition [14]. The magnetic permeability is therefore expected to change significantly during operation, influencing the skin-depth in $\mathrm{AC}$ measurements and subsequently undermining the stability of the impedance.

The skin effect must therefore be suppressed in order to achieve stable measurements in ferromagnetic materials. Figure 1 shows the current distributions for two illustrative cases at a range of frequencies. At very low, quasi-DC frequencies the skin depth will be large and therefore the current penetration depth will be limited by geometry instead of the skin effect. In the illustrations of Fig. 1, as the skin depth increases, eventually the current penetration will be limited by (a) the component thickness or (b) the electrode separation, the current distribution will tend to the DC-case. The frequency at which the skin depth equals the current limiting dimension, $a$, will be referred to as the transition frequency, $f_{T}$.

$a \equiv \frac{1}{\sqrt{f_{T} \pi \mu \sigma}}$,

or equivalently,

$f_{T} \equiv \frac{1}{a^{2} \pi \sigma \mu}$.

The transition frequency divides the AC and DC-like behaviour with a transitional regime. At low, 'quasi-DC' frequencies below the transition frequency the current penetration will no longer be dictated by the skin effect but rather geometry and therefore tend to the DC current distribution. It will therefore become increasingly insensitive to the changes in magnetic permeability that would otherwise undermine the measurement stability. At high frequencies, above the transition frequency, the current penetration does not penetrate deep enough to be influenced by geometry resulting in $\mathrm{AC}$ behaviour. In order to suppress the skin-effect the inspection frequency should be below the transition frequency; the lower the frequency the more it will tend to the DC case and the less remnant sensitivity will remain.

There is a distinct advantage of using very low frequency $\mathrm{AC}$ as opposed to $\mathrm{DC}$, which is inherently insensitive to the skin effect. For equivalent current magnitudes AC measurements have a greater signal-to-noise ratio (SNR) due to the increase in resistance from the constriction of the available cross section to current flow resulting from the skin effect. However, even in the present case where the current distribution is approximately equal to the DC distribution, the SNR is superior due to the ability to utilise phase sensitive detection using lock-in amplifiers. Further, DC measurements are susceptible to spurious thermoelectric signals and significant random noise due to flicker, or ' $1 / \mathrm{f}$ ' noise, which exhibits a noise power density that is inversely proportional to frequency [16]. An example preamplifier noise density frequency spectrum is shown in Fig. 2 to illustrate this issue. The data shown is for a Stanford Research SR560, this preamplifier was not used in this study but is included as illustrative of the performance of a commercially available preamplifier. Typically injection currents of the order of $10 \mathrm{~A}$ must be used to produce DC voltage differences that can be measured with a sufficient signal-to-noise ratio, especially on larger components $[1,17]$. An alternative approach has been proposed, whereby low frequency AC measurements are utilised. AC measurements allow phase-sensitive detection and with increasing frequency a reduction in flicker noise. With reference to Fig. 2 , in this case increasing the inspection frequency from 1 to $10 \mathrm{~Hz}$ reduces the noise density from 30 to $5.5 \mathrm{nV} / \sqrt{ } \mathrm{Hz}$. As the noise is random, a 5.5-fold reduction of the noise density permits a 30 -fold reduction in measurement energy consumption (either power or averaging time), illustrating the benefit of adopting as high a frequency as possible. Typically, at quasi-DC frequencies $100 \mathrm{~mA}$ is sufficient to provide adequate SNR's.

A compromise is a quasi-DC measurement, the inspection frequency should be as low as possible in order to suppress the skin effect, but should also be sufficiently high to reduce flicker noise. Unfortunately, quasi-DC readings will only tend towards a DC-asymptote and some remnant sensitivity to changes in the skin effect will result.

This paper proposes a correction of the spurious changes in the resistive real part of impedance by using the phase angle, a usually superfluous by-product of the measurement. There is a unique relationship between magnitude and phase at a given frequency which allows for conversion between the two, the increase in resistance due to the skin effect can therefore be approximated from the phase measurement allowing for it to be compensated and permitting recovery of the DCasymptotic resistance.

The correction has been developed for the purpose of creep strain measurements; changes in the skin depth will 
(a)

i)

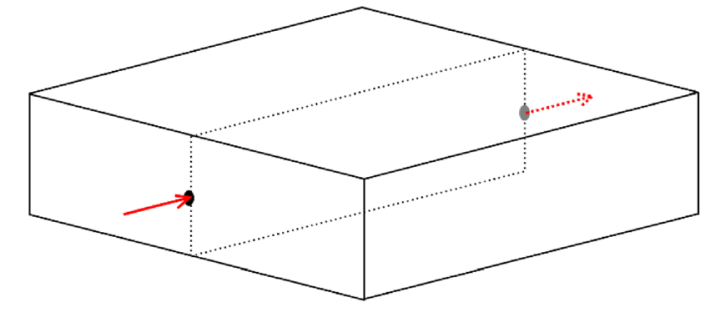

ii) $f / f_{T}=0.1$

iii) $f / f_{T}=1$
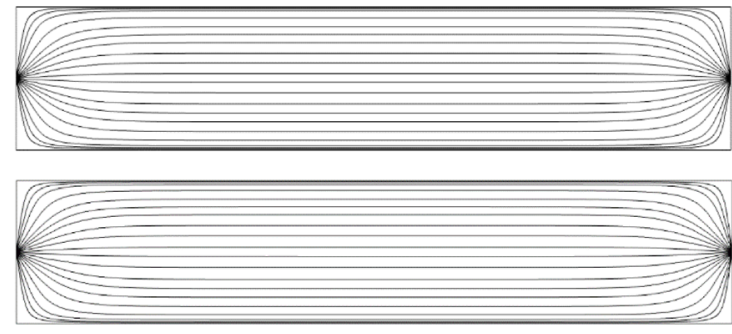

iv) $f / f_{T}=10$

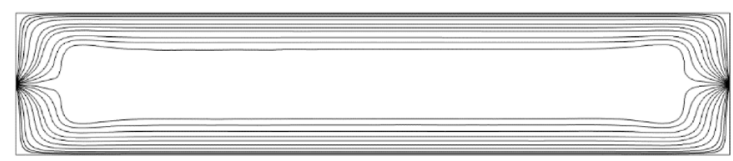

v) $f / f_{T}=100$

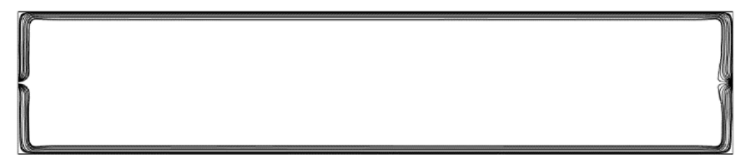

vi)

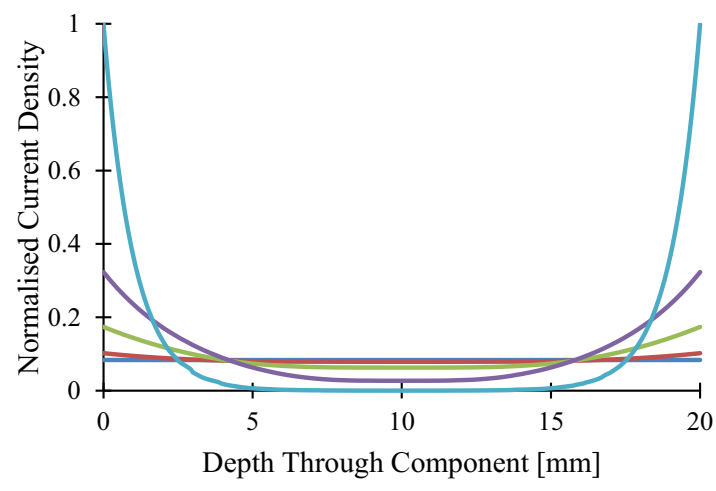

$1+1$
Normalised Frequency $=0.1$

3

Fig. 1 Finite element simulation results obtained using Comsol [15]. Current distributions in a $100 \mathrm{~mm} \times 100 \mathrm{~mm} \times 20 \mathrm{~mm}$ block with relative magnetic permeability of 1 and conductivity of $100 \%$ IACS. Column a current is injected from the centre of one edge to the other. Column b current is injected between two points separated by $10 \mathrm{~mm}$ on

invariably arise in these ferromagnetic components due to changes in stress, temperature and thermal ageing. The correction is however potentially more widely applicable, as will be illustrated by demonstrating its application to the canonical case of a conducting rod. (b)
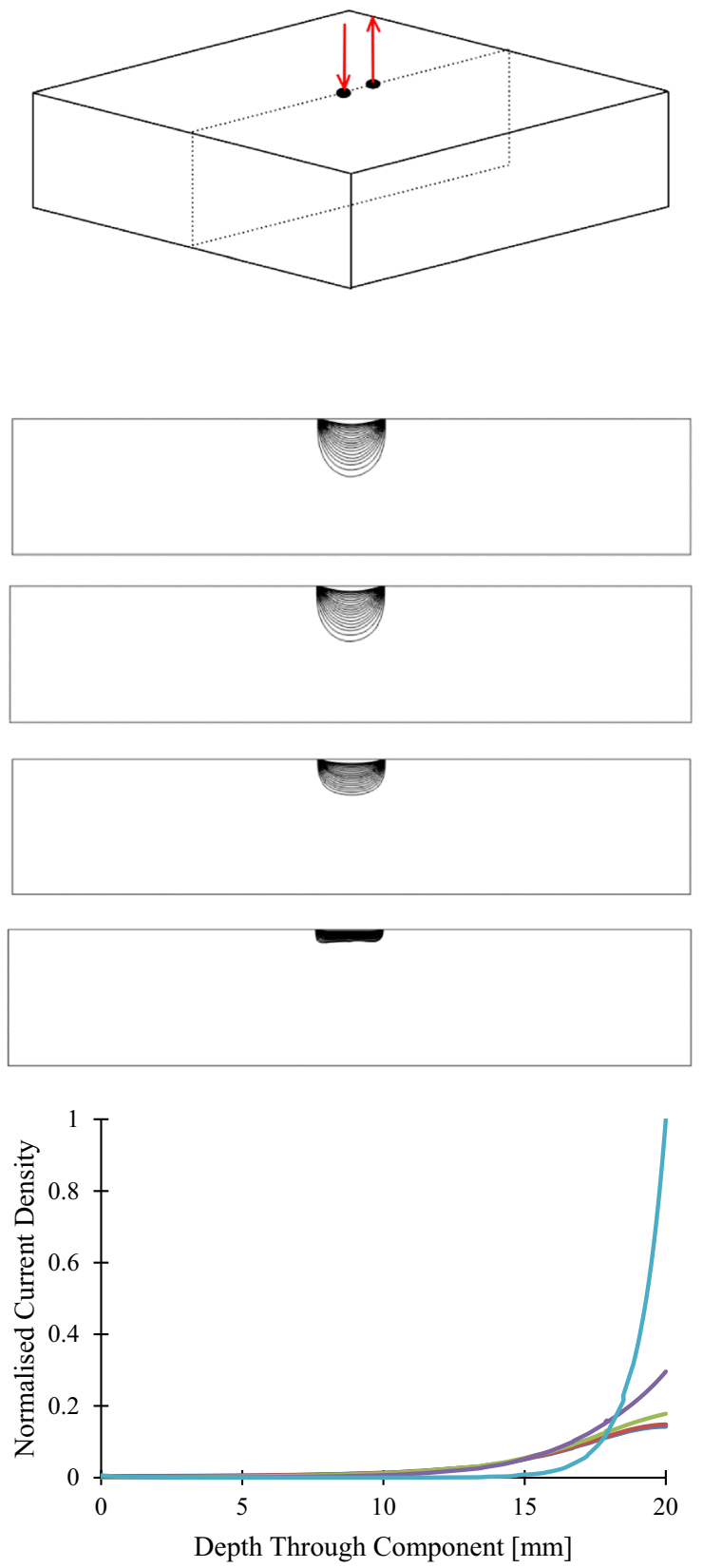

$\infty$ the surface of the component. (i) shows an illustration of the two cases. (ii)-(v) Streamlines show the current path for a range of frequencies. (vi) The current density is plotted as a function of distance from bottom of component. The current density is normalised to its maximum (at the highest inspection frequency)

A prerequisite for the correction to be applicable is that the system is minimum phase and it is shown that both the conducting rod and a conducting half space (approximating the creep problem) satisfy this requirement. An ad-hoc approximation is then presented which provides a practical 


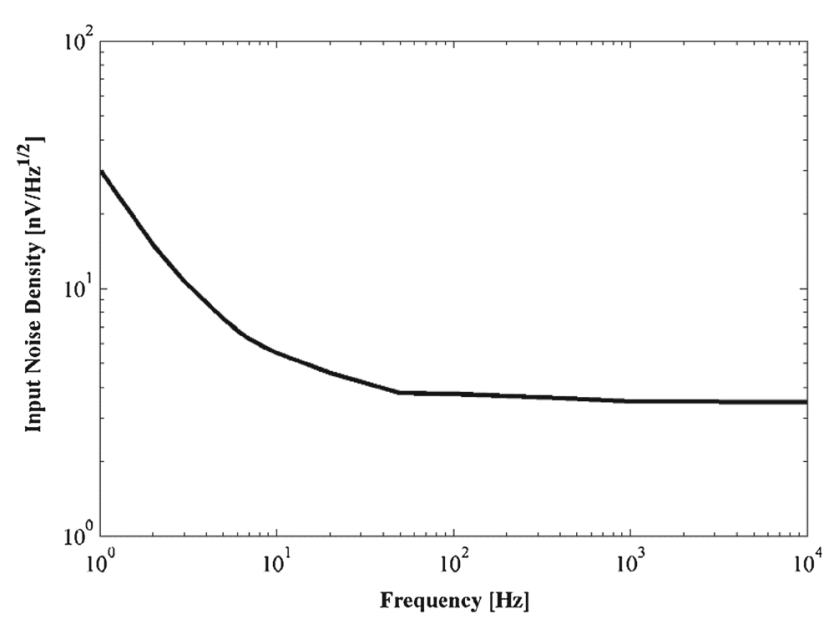

Fig. 2 Noise performance of the Stanford Research Systems SR560 low-noise preamplifier [18]. This is not the preamplifier used in the experimental work of this paper but is included as a representative example

means of implementing the correction. The correction is demonstrated experimentally on a room temperature tensile test component, with the magnetic permeability deliberately manipulated by applying elastic strain.

\section{Minimum Phase Systems}

Two potential drop measurement arrangements are shown in Fig. 3, (a) a slender conducting rod and (b) a square electrode configuration on the surface of a conducting half-space. The former is included in this paper for illustrative purposes as a familiar application (the rod behaviour will tend towards that of a wire as the normalised diameter decreases) and the latter is the application of particular interest.

The two configurations shown in Fig. 3 have exact analytical solutions for the frequency dependent complex impedance, as will be discussed in more detail in Sect. 2 . The magnitude and phase responses are shown in Fig. 4. At quasi-DC frequencies, in the case of the slender rod the current will be distributed uniformly over the whole cross section of radius, $a_{r}$, and in the case of the square electrode configuration on a conducting half-space, the current will not penetrate far beyond half an electrode separation, $a_{e} / 2$. The limiting dimensions dictating the transition frequency of Eqs. 2 and 3 are therefore $a_{r}$ and $a_{e} / 2$ respectively.

Two distinct regimes are evident, high-frequency $\mathrm{AC}$ and low-frequency DC asymptotic behaviour, joined by a transitional regime that is of interest in the present paper. At low frequencies the magnitude tends to the DC-asymptotic value and 0 phase while at high frequencies, the magnitude of the complex impedance becomes approximately proportional to the square root of frequency while the phase asymptotically approaches $\pi / 4$.

Minimum phase systems are causal and stable [19]. The feature of minimum phase systems that will be exploited in this paper is that the relationship between magnitude and
Fig. 3 Schematics showing two potential drop measurement arrangements, a a slender rod and $\mathbf{b}$ a square electrode configuration on a conducting half-space

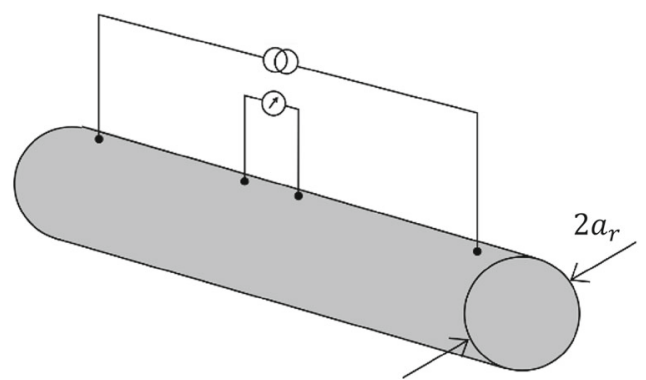

(a) Conducting Rod

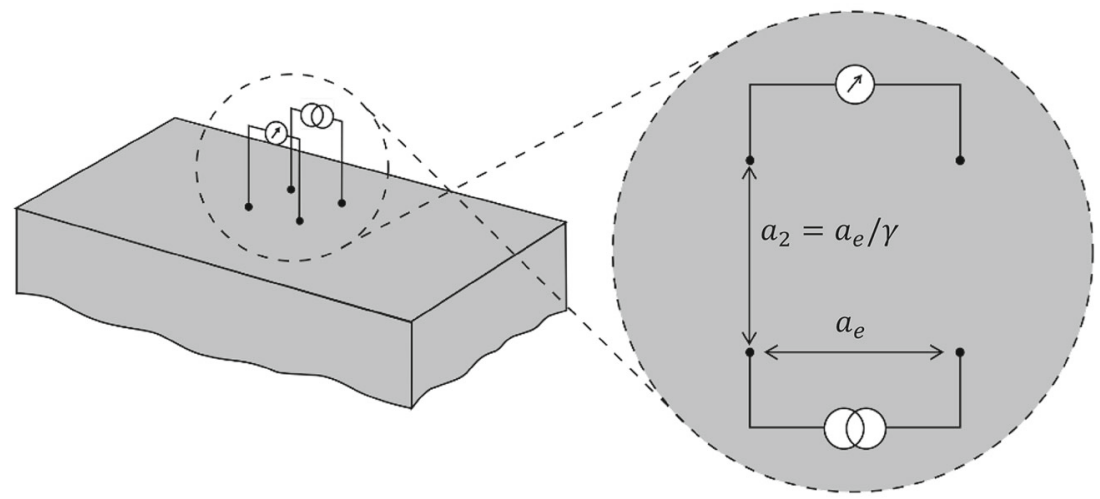

(b) Square Electrode Configuration on a Conducting Half-space 
Fig. 4 Impedance frequency response of the two measurement configurations shown in Fig. 3
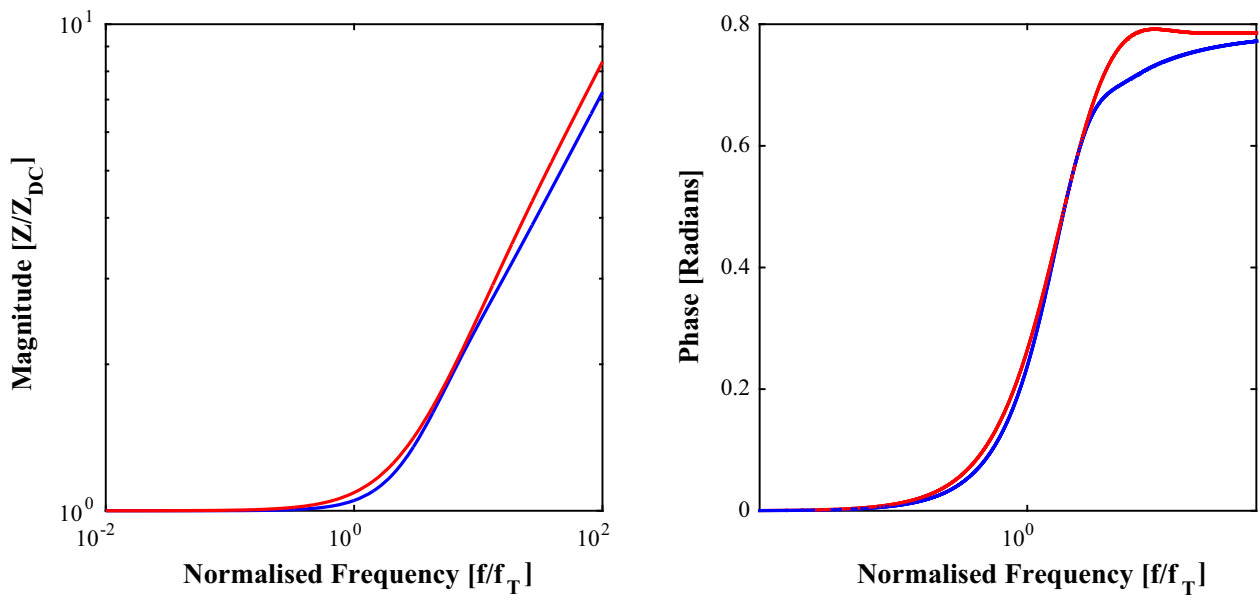

- Giacolletto - Cylindrical Conducting Rod

Bowler - Square configuration of electrodes on conducting half-space

phase provides unique solutions. As quasi-DC potential drop readings are minimum phase it means that spurious changes in the real part arising from the skin effect can be estimated and therefore compensated using the phase angle.

A useful property of minimum phase systems is that the phase and the natural logarithm of the magnitude form a Hilbert-transform pair [19]

$\arg [K(\omega)]=\varphi(\omega)=-\mathcal{H}\{\ln (|K(\omega)|)\}$

where $\mathcal{H}$ is the Hilbert transform and $K(\omega)$ is the transfer function of the system.

The Hilbert transform can be used to show that the impedance resulting from $\mathrm{AC}$ potential drop measurements are also minimum phase. While this is only shown for two geometries in this paper the authors believe this is true for alternating current potential drop (ACPD) measurements in general. This feature can be exploited to infer the magnitude change by using a Hilbert transform of the phase, thereby recovering the DC-asymptotic value from measurements at higher frequencies. The phase change arising from the skineffect can therefore be used to quantify and compensate spurious changes in the real, resistive part.

The two potential drop measurement arrangements discussed in the introduction have established analytical solutions enabling the numerical demonstration that they are minimum phase using the Hilbert transform. The solutions also provide an analytical basis for the validation of the adhoc phase based correction.

The frequency response of impedance (without the selfimpedance term) in a slender rod conductor is given by Giacoletto [20]

$$
\frac{Z(k)}{R_{D C}}=\frac{k a_{r} J_{0}\left(k a_{r}\right)}{2 J_{1}\left(k a_{r}\right)},
$$

where $k$ is equal to $(1-i) / \delta$ and $a_{r}$ is the rod radius. $J_{0}$ and $J_{1}$ are zero- and first-order Bessel function of the first kind, respectively.

The frequency response of a rectangular electrode configuration on the surface of a conducting half space is derived from the series expansion approximation provided by Bowler [21].

$$
\frac{Z(k)}{R_{D C}}=1+\frac{\frac{1}{\sqrt{1+1 / \gamma^{2}}} \sum_{n=1}^{\infty} \frac{\left(-i k a_{e} / 2 \gamma \sqrt{1+1 / \gamma^{2}}\right)^{n+1}}{n(n+1) !}-\sum_{n=1}^{\infty} \frac{\left(-i k a_{e} / 2 \gamma\right)^{n+1}}{n(n+1) !}}{\left(1-\frac{1}{\sqrt{1+1 / \gamma^{2}}}\right)},
$$

where $a_{e}$ is the electrode separation between the current injecting electrodes and $\gamma$ is the aspect ratio of the rectangle formed by the electrodes $a_{2}=a_{e} / \gamma$.

Using Eq. 4 it can be shown that impedance calculated for these potential drop measurements are minimum phase, as illustrated by the agreement of Fig. 5 .

It can be concluded that the Hilbert transform of the phase could be used to correct the influence of the skineffect on the magnitude and therefore also the resistive part of the impedance. In practise, however, this process is considered to be too involved and a simpler 'engineering' solution is sought. In the next section, a generalised ad-hoc approximation is presented which is applicable to a range of measurement configurations and may be adopted without explicit knowledge of the impedance response.

\section{An Ad-Hoc Phase Based Correction for the Skin Effect}

The following description of an ad-hoc correction is described assuming the NDE application of interest, the square electrode 


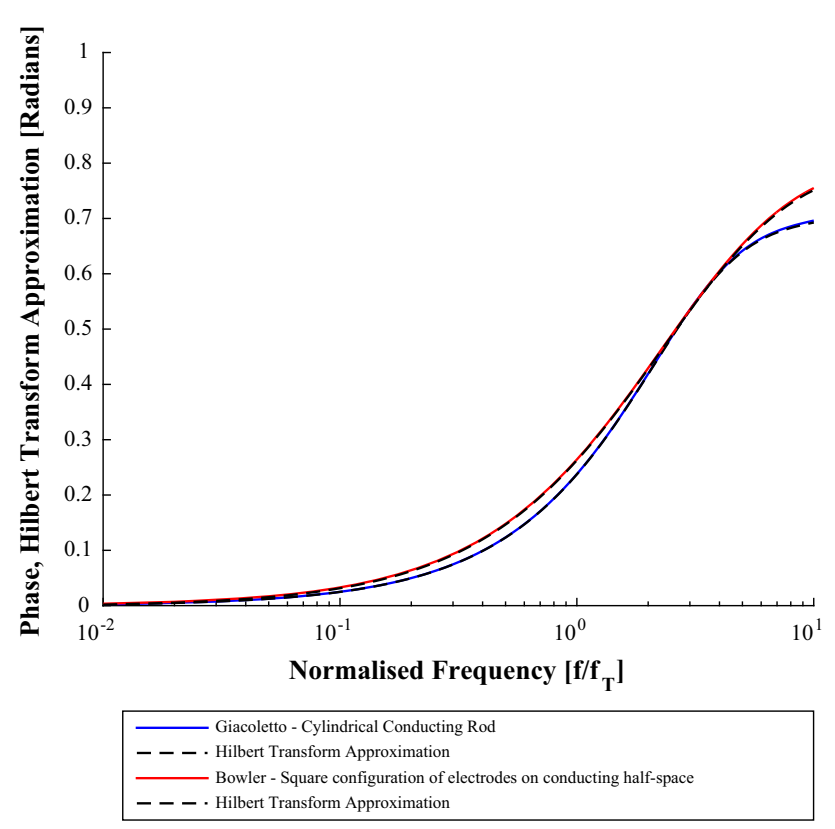

Fig. 5 Phase calculated using Eqs. 5 and 6. Hilbert transform approximation of phase using Eq. 4. The good agreement indicates that the impedance is minimum phase

configuration on a conducting half space, it is however believed that the approach is more broadly applicable, as will be demonstrated using the conducting rod as an example.

\subsection{Asymptote}

The DC asymptotic resistance for a square electrode configuration in a 'thick' (conducting half space) component $[7,22]$.

$R_{D C \text { thick }}=\frac{\rho}{\pi} \frac{1}{a_{e}}\left[1-\frac{1}{\sqrt{2}}\right]$
It is noted by Sposito et al. [10] that this has the general form:

$$
R_{D C \text { thick }}=\frac{\rho}{\pi} k_{1}
$$

where $k_{1}$ is a constant determined by the electrode configuration.

\subsection{AC Asymptote: The Reduced-Thickness Model}

A simplification that has been previously employed to account for the skin effect in AC potential drop measurements is to assume that the current distribution is that of a uniformly distributed current but constrained to a depth equal to the skin depth. This observation is convenient but not coincidental as explained by Sposito et al. [23]. The result is that at frequencies considered to be $\mathrm{AC}$, the potential drop is equivalent to a DC distribution in a thin plate of thickness $T=\delta=\frac{1}{\sqrt{ } \pi f \mu \rho}$, as illustrated in the schematic of Fig. 6 .

The 'thin' component DC resistance for a square electrode configuration is again provided by Madhi [24]:

$R_{D C \text { thin }}=\frac{\rho}{\pi} \frac{\ln (2)}{2 T}$.

Using the reduced thickness model the AC resistance asymptote can be established,

$R_{A C \text { thick }}=\frac{\rho}{\pi} \frac{\ln (2)}{2 \delta}$.

Again, this result can be generalised to

$R_{A C \text { thick }}=\frac{\rho}{\pi} \frac{1}{\delta} k_{2}$,

where $k_{2}$ is a constant determined by the electrode configuration and component geometry.
Fig. 6 a Schematic current distribution resulting from an $\mathrm{AC}$ injected current and $\mathbf{b}$ the equivalent reduced thickness model

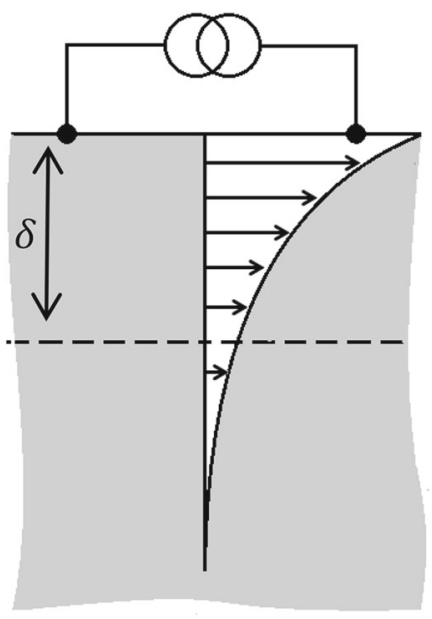

(a) AC Current Distribution

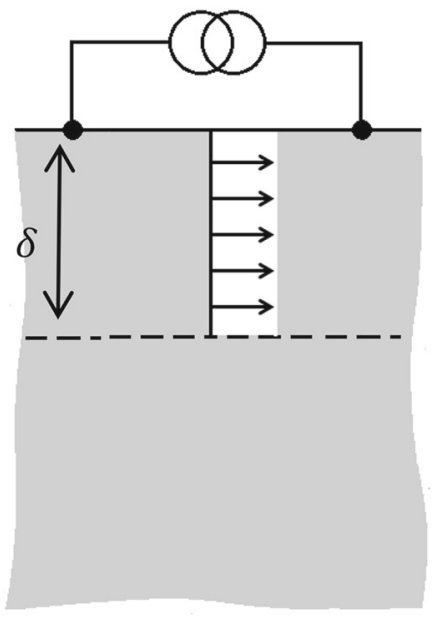

(b) Reduced Thickness Model 


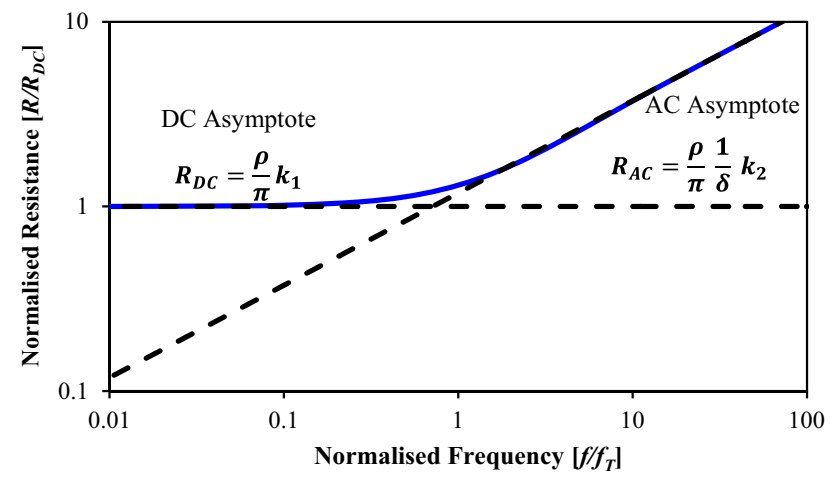

Fig. 7 Analytical solution for normalised resistance versus normalised frequency from Eq. (6). Asymptotes plotted from Eqs. 7 and 10 7.

The two asymptotes are therefore defined as shown in Fig.

\subsection{Transition Approximation}

Sposito et al. suggests the following approximation for the resistance at a given frequency to account for the transition from the $\mathrm{AC}$ to the $\mathrm{DC}$ regimes [10],

$R=\left(R_{D C}^{\beta}+R_{A C}^{\beta}\right)^{1 / \beta}$,

where $\beta$ is a parameter which described the shape of the transition between asymptotes. Using Eqs. 8 and 11 this can be written:

$R=R_{D C}\left[1+\left(\frac{k_{2}}{k_{1}} \frac{1}{\delta}\right)^{\beta}\right]^{1 / \beta}$.

Using the previous definitions of the skin depth and transition frequency Eq. 3 then:

$\frac{1}{\delta}=\frac{1}{a} \sqrt{\frac{f}{f_{T}}}$

Combining Eqs. 13 and 14 gives,

$R=R_{D C}\left[1+\left(\frac{k_{2}}{k_{1}} \frac{1}{a} \sqrt{\frac{f}{f_{T}}}\right)^{\beta}\right]^{1 / \beta}$.

The term $\frac{k_{2}}{k_{1}} \frac{1}{a}$ is specific to the geometry. Introducing a new corner frequency, defined as

$f_{C}=f_{T} /\left(\frac{k_{2}}{k_{1}} \frac{1}{a}\right)^{2}$

allows the simplification and generalisation of Eq. 15. This newly defined corner frequency provides an improved refer- ence point which is defined in terms of the transition from $\mathrm{DC}$ to $\mathrm{AC}$ regime as opposed to physical dimensions.

$R=R_{D C}\left[1+{\frac{f}{f_{C}}}^{\frac{\beta}{2}}\right]^{1 / \beta}$

$\beta$ can then be found for the given geometry by regression to a known solution or empirical data.

\subsection{Phase Approximation of Skin Effect}

The approximation of Eq. 17 is of limited use without a good estimate of the corner frequency, a datum that moves with the skin depth. In order to avoid this requirement it is suggested that the phase, a usually neglected by-product of such measurements, is utilised. A little physical insight offers a general trend for resistance, $R$, and reactance, $X$, (and hence phase) over a range of normalised frequencies. The relationship between phase and normalised frequency can therefore be used to eliminate the normalised frequency in Eq. 17.

In the quasi-DC regime the resistance clearly tends towards the DC resistance, only increasing when the skin effect becomes significant. The reactance however is zero at DC and initially increases proportionally with frequency. At higher frequencies where the penetration depth is controlled by the skin effect both the resistance and reactance increase with the square root of frequency. The previously defined corner frequency fulfils the following relationship;

$$
\begin{aligned}
& \left.\begin{array}{l}
f \ll f_{C} \\
R=R_{D C} \\
X=R_{D C}\left(\frac{f}{f_{C}}\right) \\
f \gg f_{C} \\
R=R_{D C}\left(\frac{f}{f_{C}}\right)^{\frac{1}{2}} \\
X=R_{D C}\left(\frac{f}{f_{C}}\right)^{\frac{1}{2}}
\end{array}\right\} R X=R_{D C}^{2}\left(\frac{f}{f_{C}}\right) \\
& X\left(\frac{f}{f_{C}}\right)
\end{aligned}
$$

$R X$ is therefore the same in both the low and high frequency regimes. This can be verified using the analytical result from Bowler, Eq. 6. In this case the geometric constant relating the transition and corner frequency is known from Eqs. 7, 10 and 16, $f_{C}=f_{T} / 1.4$ allowing the illustration in Fig. 8.

This allows the phase relationship to be established from the usual convention,

$\tan \varphi=\frac{X}{R}$ 


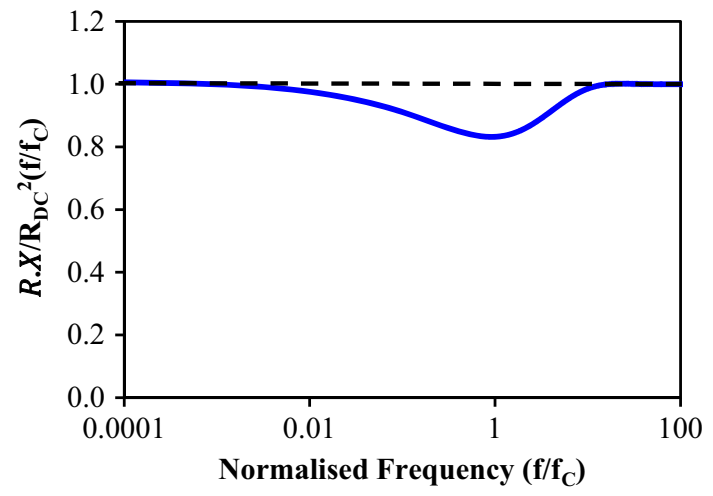

Fig. 8 Illustration that the geometric mean of the resistance and the reactance is approximately equal to the sought DC asymptote of the resistance over the whole frequency range

and from Eqs. 17 and 18,

$\tan \varphi=\frac{\frac{f}{f_{C}}}{\left[1+\frac{f}{f_{C}}{ }^{\frac{\beta}{2}}\right]^{1 / \beta}}$

Combining Eqs. 17 and 20 to eliminate $\frac{f}{f_{C}}$ yields,

$\frac{R}{R_{D C}}=\frac{1}{\left(1-(\tan \varphi)^{\frac{\beta}{2}}\right)^{1 / \beta}}$

$\beta$ can be found using regression to a known analytical solution or empirical data. The solution of Eq. 6 from Bowler [21] will be used in this case.

\subsection{Limitation of a Phase Based Correction}

It is important to note that the correction provided here only describe the unique relationship between the resistance and phase arising from the skin effect. The assumed relationship may be violated by contributions from other unaccounted influences, for example from inductive coupling and signal shaping electronics. Inductive coupling between the injection and sensing loops would introduce an additional reactance that increases proportionally with frequency, and the additional reactive part would undermine the correction. Fortunately at the near-DC frequencies relevant to the proposed correction inductive effects will be minimal provided that the usual practical measures are enacted to minimise inductive coupling. A suggested experimental check that inductive effects are kept below an acceptable threshold is to increase the frequency and ensure that the phase angle tends to $45^{\circ}$. For a more detailed discussion of this effect see $[21,25]$. Additionally, a practical note is that any phase and magnitude changes resulting from signal conditioning must be taken into account. The transfer functions of most filters are widely known and can be easily found in literature [26].

In order for the methodology outlined in this paper to be of use then it is required that the phase must have reasonable sensitivity to changing frequency in the region where the resistive component of the complex impedance perceivably deviates from its DC asymptote. As already noted, at higher frequencies both the reactance and resistance tend to the same value and therefore the phase tends to $\pi / 4$ radians. Plotting the phase against frequency shows that the sensitive range is limited to the close vicinity of the transition frequency as shown in Fig. 4. This limitation is compounded by the asymptotic behaviour of Eq. 21, as $\varphi \rightarrow \pi / 4, R / R_{D C} \rightarrow \infty$ Therefore, when finding best-fitting values of $\beta$ large errors in the correction are introduced as $\varphi \rightarrow \pi / 4$. To mitigate this limitation the approximation is adjusted by introducing an arbitrary second parameter, $A$. The parameter has no physical significance but performs the mathematical function of changing the asymptotic behaviour of the correction allowing for correction up to $\varphi=\pi / 4$. While this circumvents the mathematical limit of Eq. 21, the physical limitation of sensitivity to below $\pi / 4$ is still present.

$$
\frac{R}{R_{D C}}=\frac{1}{\left(1-A \tan ^{\frac{\beta}{2}} \phi\right)^{1 / \beta}}
$$

Figure 9 illustrates the varying degrees of success of Eqs. 21 and 22. While the simpler formulation of Eq. 21 can be seen to be very good up to approximately $35^{\circ}\left(f / f_{T}=1\right)$, its limiting asymptotic behaviour means that it is poor when fitted up to $45^{\circ}$. The suggested modification of Eq. 22 is seen to have excellent agreement with the analytical solution. The values of $\beta$ found in Fig. 9 are consistent with the findings of Sposito et al. at $\beta \approx 3$ [10].

The case of a square electrode configuration on a conducting half-space has been assumed to illustrate the process, however, the previous arguments are common to all geometries, but the parameters $A$ and $\beta$ are geometry specific. Figure 10 shows how this process can be applied to the case of the conducting rod as demonstrated using the solution in Eq. 5 provided by Giacoletto [20].

An important consideration for utilising the ad-hoc correction outlined in this paper is the geometry specific fitting parameters. In the case of the square electrode configuration measurement the intended application is for strain measurement. As the component deforms, changes in the electrode configuration aspect ratio, $\gamma$, are expected; with reference to Eq. 6, an accompanying change in impedance will result and with it the best fitting parameters. Impedance values were calculated from Eq. 6 for two aspect ratios, $\gamma=1$ and $\gamma=1.1$, which may result from strain accumulation in the 
Fig. 9 Approximated and exact relationship between phase angle and resistance for a square configuration of electrodes on a conducting half space. Best fitting parameters are shown for each approximation in the table

Fig. 10 Approximate and exact relationship between phase angle and resistance for a conducting rod. Best fitting parameters are shown for each approximation in the table

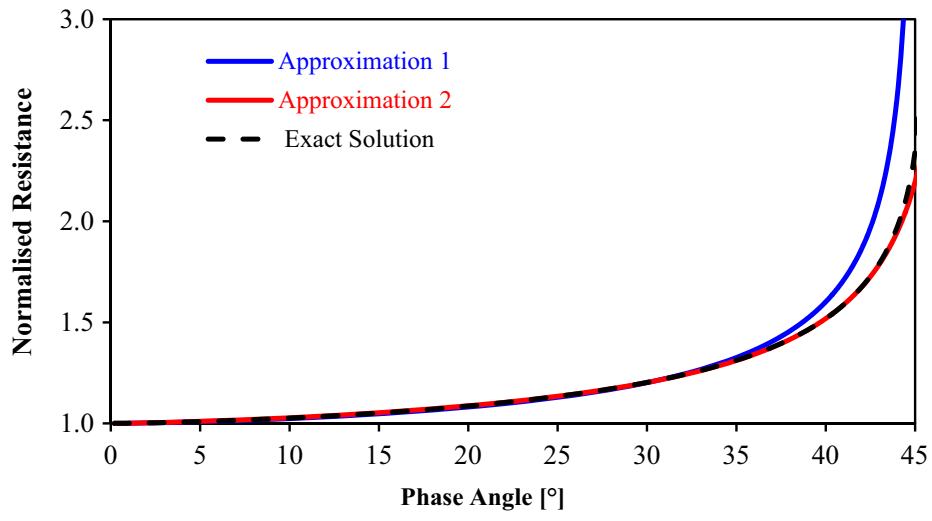

\begin{tabular}{lccl}
\hline & $\boldsymbol{A}$ & $\boldsymbol{\beta}$ & Notes \\
\hline Exact Solution & N/A & N/A & From Bowler [21] \\
Approximation 1 & N/A & 3.06 & Eq. 21: Optimised up to transition frequency, $\boldsymbol{\varphi} \approx \mathbf{3 5}^{\circ}$ \\
Approximation 2 & 0.94 & 2.92 & Eq. 22: Optimised up to twice transition frequency, \\
& & $\boldsymbol{\varphi} \approx \mathbf{4 5}^{\circ}$
\end{tabular}

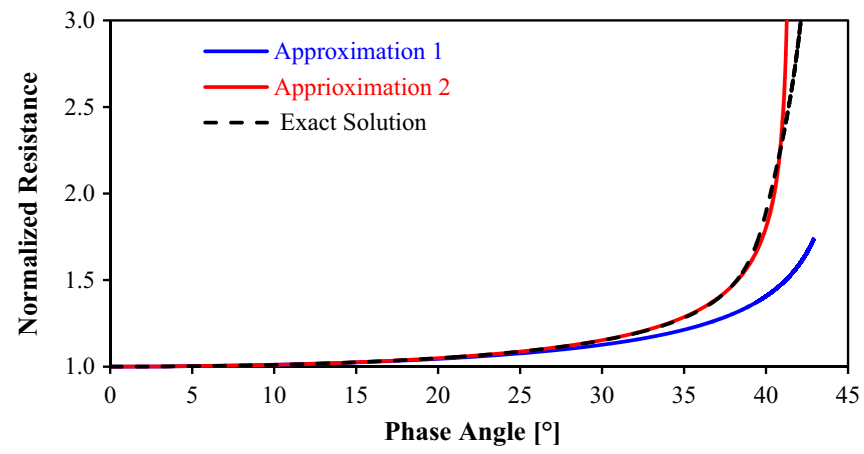

\begin{tabular}{lccl}
\hline & $\boldsymbol{A}$ & $\boldsymbol{\beta}$ & Notes \\
\hline Exact Solution & N/A & N/A & From Giacoletto [20] \\
$\begin{array}{l}\text { Approximation } \\
\mathbf{1}\end{array}$ & N/A & 3.73 & Eq. 21: Optimised up to transition frequency, $\boldsymbol{\varphi} \approx \mathbf{1 4}^{\circ}$ \\
$\begin{array}{l}\text { Approximation } \\
\mathbf{3}\end{array}$ & 1.13 & 3.96 & Eq. 22: Optimised up to twice transition frequency, $\boldsymbol{\varphi} \approx \mathbf{2 4}^{\circ}$ \\
\hline
\end{tabular}

test component. Table 1 shows the best fitting parameters, the changes in parameters are modest.

\subsection{Summary of Proposed Correction Procedure}

The proposed procedure for the correction of the skin effect is summarised here:

1. Take resistance and phase readings for a range of frequencies covering quasi-DC, transition and $\mathrm{AC}$ frequencies.

2. Correct values for any signal shaping that has occurred (usually low or high pass filters).

3. Validate that the phase angle tends to $\pi / 4$ at higher frequencies to ensure that inductive effects are not domi- nating. Frequencies where the phase exceeds $\pi / 4$ should not be used.

4. Fit values to Eq. 21 or Eq. 22; find best fitting parameters $A$ and $\beta$.

5. Carry out measurements at the chosen frequency, benefitting from improved noise performance. Use Eq. 21 or 22 to correct for the skin effect.

6. Significant distortion of the measurement geometry will reduce the accuracy of the correction.

\section{Experimental Demonstration}

To demonstrate the proposed correction the magnetic permeability of a test component was manipulated using piezo- 
Table 1 Best fitting parameters are shown for different approximations and for electrode configurations that are square, $\gamma=1$, and rectangular with an aspect ratio of $\gamma=1.1$

\begin{tabular}{|c|c|c|c|c|c|}
\hline & \multicolumn{2}{|c|}{$\gamma=1$} & \multicolumn{2}{|c|}{$\gamma=1.1$} & \multirow[t]{2}{*}{ Notes } \\
\hline & $A$ & $\beta$ & $A$ & $\beta$ & \\
\hline Approximation 1 & N/A & 3.06 & N/A & 3.04 & Eq. 21: optimised up to transition frequency, $\phi \approx 35^{\circ}$ \\
\hline Approximation 2 & 0.94 & 2.92 & 0.93 & 2.85 & $\begin{array}{l}\text { Eq. 22: optimised up to twice transition frequency } \\
\phi \approx 45^{\circ}\end{array}$ \\
\hline
\end{tabular}

Fig. 11 Normalised resistance against inspection frequency for different applied stresses. a and $\mathbf{b}$ are uncorrected while $\mathbf{c}$ and $\mathbf{d}$ have been corrected using the asymptotic DC phase correction. $\mathbf{a}$ and $\mathbf{c}$ are measurements taken in the parallel direction while $\mathbf{b}$ and $\mathbf{d}$ are in the normal direction. (For interpretation of the references to colour in this figure legend, the reader is referred to the web version of this article)
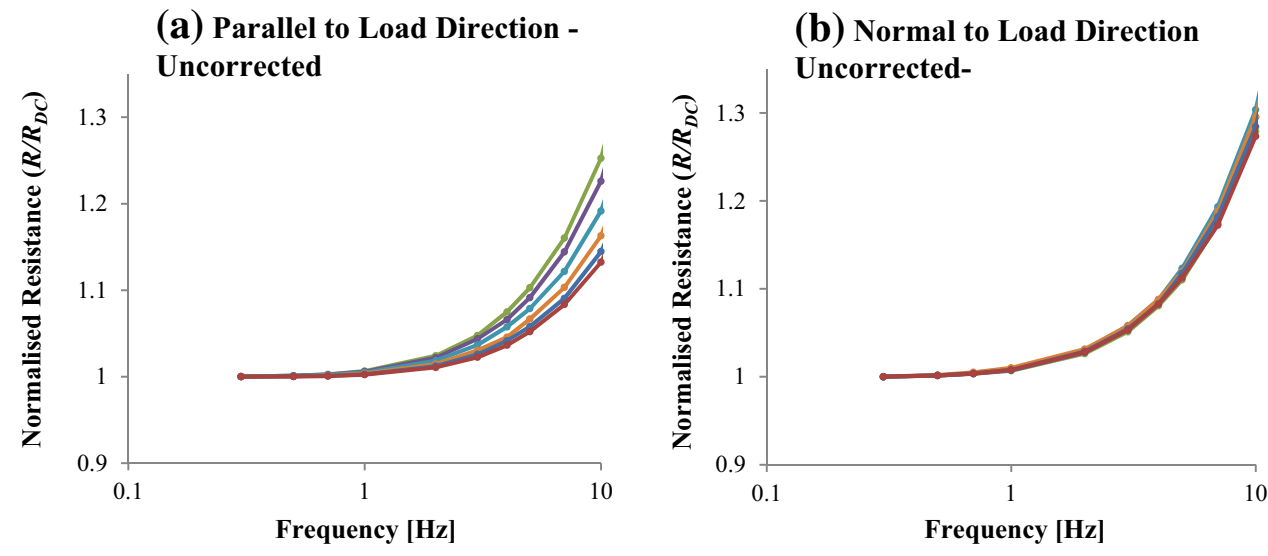

(c) Parallel to Load Direction Corrected
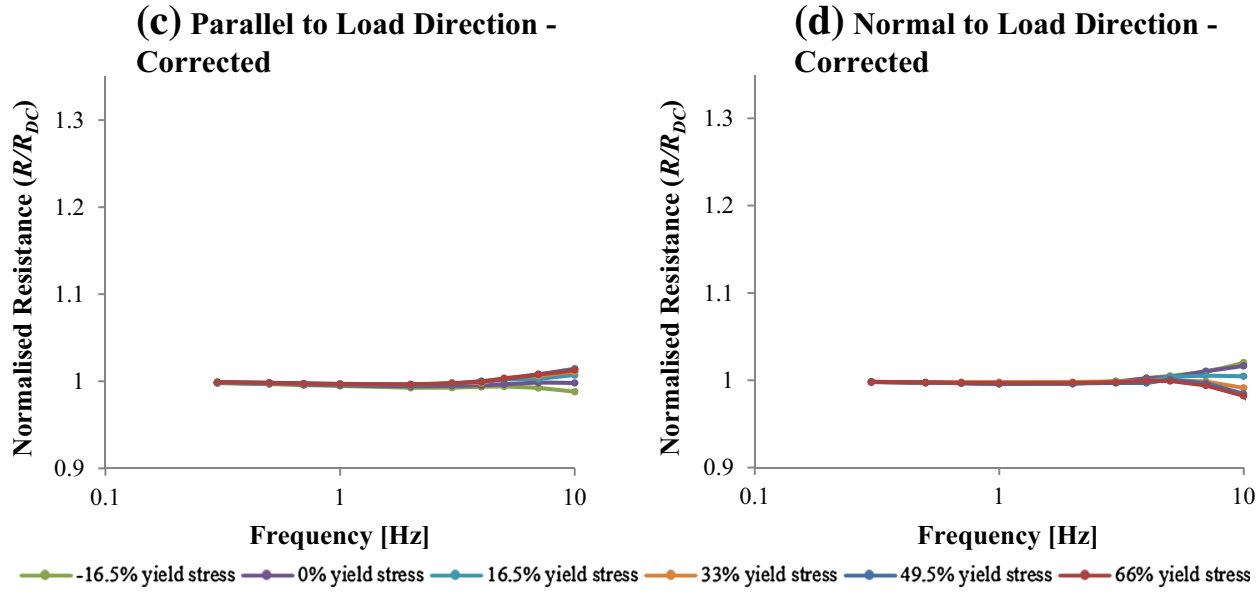

magnetism. Applying different elastic loads will alter the permeability in a controlled and simple way allowing the correction to be validated experimentally. The small elastic strains will have a negligible influence on the DC-asymptotic value of the resistance compared to the influence of the skin effect at higher frequencies.

A tensile test specimen of S275 steel with cross section $75 \mathrm{~mm} \times 24 \mathrm{~mm}$ was prepared with conventional strain gauges and the load cells integrated into the tensile test machine to measure stress. In addition to this, a bi-directional square configuration potential drop sensor of $5 \mathrm{~mm}$ electrode separation was installed by welding electrodes to the surface of the component. An injected current of $300 \mathrm{~mA}$ was utilised.
A series of loads of $-100,0,100,200,300$ and $400 \mathrm{kN}$ were applied, this equates to stresses of $-16.5,0,16.5,33$, 49.5 and $66 \%$ of the yield stress. At each stress potential drop measurements were taken in both directions with inspection frequencies of $0.3,0.5,0.7,1,2,3,4,5,7,10 \mathrm{~Hz}$.

Figure $11 \mathrm{a}$ and $\mathrm{b}$ shows the results from the experiment. Resistance values have been normalised to their DC value, taken here to be the $0.3 \mathrm{~Hz}$ measurement; at ever decreasing frequencies the resistances tend to the DC-asymptote as expected. The frequency independence at low frequencies shows that $0.3 \mathrm{~Hz}$ is within the quasi-DC regime and therefore considered independent of the skin-effect. When using this technique for creep strain measurements it is intended that the changes in the DC asymptote resulting from resistiv- 
Table 2 Best fitting parameters for correction of experimental data

\begin{tabular}{llll}
\hline & $\varphi_{\max }\left[^{\circ}\right]$ & $A$ & $\beta$ \\
\hline Parallel to loading direction & 32 & 0.99 & 2.92 \\
Normal to loading direction & 37 & 0.91 & 3.00 \\
\hline
\end{tabular}

ity changes and strain will provide information on the creep state.

In this demonstration the influence of the elastic load has only a marginal influence on the DC- resistance. The strain is limited to only $\sim 0.08 \%$ and the effect of piezoresistivity was seen to be minimal. Temperatures were monitored to be within $1{ }^{\circ} \mathrm{C}$. The combined effects provided a resistance range of $0.6 \%$ over the stress range. Changes in the DCasymptotic value are not evident.

At higher frequencies the frequency dependence resulting from the skin effect is clear. As the inspection frequency increases the skin depth is reduced, minimising the effective area the current can flow through and therefore the resistance rises. Figure 11a shows most clearly the symptomatic changes in resistance resulting from the changing skin depth caused by varying elastic load. As the elastic load is increased, the magnetic permeability reduces, the skin depth is therefore increased permitting a greater available cross section for current flow and therefore resistance falls. Equivalently, this process can be described in terms of transition frequency; as the permeability reduces, the transition frequency increases according to Eq. 3, causing a shift in the resistance frequency response. Figure $11 \mathrm{~b}$ appears to show less sensitivity to changes in elastic load. It is important to remember that magnetic permeability should be considered a tensor [14] and therefore the change in the skin effect is different in the orthogonal directions.

By measuring the phase angle the influence of the skin effect can be estimated and corrected for using the procedure given in Sect. 3.5. The parameters $A$ and $\beta$ were found by regression to minimise the deviation from $R_{D C}$. The best fitting parameters are shown in Fig. 11 and are consistent with the expectation that $A \approx 1$ and $\beta \approx 3$. Also displayed is the maximum phase angle measured in each configuration. The discrepancy between values is due to the initially imperfect electrode placement resulting in an effective aspect ratio of the electrode square (Table 2).

\section{Discussion}

The experimental demonstration in this paper is intended only as an example of one of the many different parameters that may cause a change in magnetic permeability and therefore skin depth. The use of elastic strain is a means of conveniently and easily controlling the magnetic permeability and demonstrating the potential uncertainty arising from taking AC measurements in ferromagnetic materials. It has been shown that by using the proposed phase based correction the DC-asymptotic value can be successfully recovered from measurements taken at quasi-DC frequencies where the uncorrected skin effect would adversely influence the measurement accuracy.

The result of this correction is that it allows some flexibility in the inspection parameters. The conservatism that would usually be required to ensure that inspection was always fully within the quasi-DC regime may be relaxed. It allows utilisation of higher inspection frequencies that may be necessary through instrument limitations or desirable to reduce noise. Referring back to Fig. 2, by increasing the inspection frequency from 1 to $10 \mathrm{~Hz}$ the noise density drops by a factor $\approx 5.5$ which would permit a thirty-fold reduction in measurement time or measurement power.

It may be desirable to increase the electrode separation constituting an important increase in the gauge length and effective penetration depth for strain measurements using the ACPD monitoring technique. This however has the undesirable effect of lowering the frequency at which the skin effect becomes significant. By relaxing the requirement on complete suppression of the skin effect the electrode separation can be increased.

The post-correction error must be assessed in relation to the magnitude of changes that are expected in the DC asymptotic value. The decision to utilise this correction must be considered as a compromise between the size of the postcorrection error and the benefits gained through operation at a higher frequency.

\section{Conclusions}

AC measurements have enhanced noise performance due to the implementation of phase sensitive detection and additionally performance is further improved by operating at higher frequencies where the flicker noise density is decreased. Unfortunately, AC measurements are subject to the skin effect so spurious changes in magnetic permeability will introduce a significant uncertainty which must be suppressed by reducing the inspection frequency as far as possible, forcing a compromise. It is therefore probable that the measurement will be in a regime where remnant sensitivity to the skin effect introduces measurement uncertainty; a correction is required to compensate for this potential error.

The correction was developed for the particular practical application of a creep strain sensor, a square configuration of electrodes on the surface of a thick conducting component which may be approximated as a conducting half space. Additionally, the case of a conducting rod was used as an 
example with a well-known analytical solution to illustrate the wider applicability of the correction to a range of geometries.

A prerequisite condition for the correction is that the system is minimum phase. It is demonstrated through the use of the Hilbert transform that both the conducting rod and conducting half space are minimum phase, providing justification for the presence of a correction. An ad-hoc approximation to the correction has been described which provides a practical procedure for implementing the correction.

The correction has been experimentally demonstrated on a tensile test component where the magnetic permeability is purposefully manipulated by applying a range of elastic loads. The wider applicability of this method is additionally demonstrated using the analytical model of the conducting rod.

Low-frequency AC measurements offer greatly enhanced noise performance over the DC alternative. The correction presented in this paper should be adopted in order to make use of the quasi-DC technique while suppressing possible uncertainty due to remnant sensitivity to the skin effect.

Open Access This article is distributed under the terms of the Creative Commons Attribution 4.0 International License (http://creativecomm ons.org/licenses/by/4.0/), which permits unrestricted use, distribution, and reproduction in any medium, provided you give appropriate credit to the original author(s) and the source, provide a link to the Creative Commons license, and indicate if changes were made.

\section{References}

1. Nagy, PB.: Electromagnetic nondestructive evaluation. In: Kundu, T. (ed.). Ultrason. Electromagn. NDE Struct. Mater. Charact. Eng. Biomed. Appl. CRC Press, Boca Raton, USA, p. 890 (2012)

2. McMaster, R.C.: Electric current test principles. Nondestruct. Test. Handb. 1st edn. Ronald Press, New York, pp. 35.1-35.11 (1959)

3. Saxena, A.: Electrical potential technique for monitoring subcritical crack growth at elevated temperatures. Eng. Fract. Mech. 13, 741-750 (1980). doi:10.1016/0013-7944(80)90005-3

4. Gilbey, D.M., Pearson, S.: Measurement of the length of a central or edge crack in a sheet of metal by an electrical resistance method. Ministry of Aviation, Royal Aircraft Establishment, RAE Farnborough (1966)

5. Dogan, B., Nikbin, K., Petrovski, B., Ceyhan, U., Dean, D.W.: Code of practice for high-temperature testing of weldments. Int. J. Press. Vessel Pip. 83, 784-977 (2006). doi:10.1016/j.ijpvp.2006. 08.011
6. Corcoran, J., Hooper, P., Davies, C., Nagy, P.B., Cawley, P.: Creep strain measurement using a potential drop technique. Int J Mech Sci 110, 190-200 (2016). doi:10.1016/j.ijmecsci.2016.03.015

7. Corcoran J.: Creep monitoring using permanently installed potential drop sensors. PhD Thesis, Imperial College London (2015)

8. Černý, I.: The use of DCPD method for measurement of growth of cracks in large components at normal and elevated temperatures. Eng. Fract. Mech. 71, 837-848 (2004). doi:10.1016/ S0013-7944(03)00012-2

9. Sposito G. Advances in potential drop techniques. $\mathrm{PhD}$ Thesis, Imperial College London (2009)

10. Sposito, G., Cawley, P., Nagy, P.B.: Potential drop mapping for the monitoring of corrosion or erosion. NDT\&E Int 43, 394-402 (2010). doi:10.1016/j.ndteint.2010.03.005

11. Bowler, N., Huang, Y.: Electrical conductivity measurement of metal plates using broadband eddy-current and four-point methods. Meas. Sci. Technol. 16, 2193-2200 (2005). doi:10.1088/ 0957-0233/16/11/009

12. Byeon, J.W., Kwun, S.I.: Nondestructive evaluation of thermally degraded 2. 25Cr-1Mo steel by electrical resistivity measurement. Mater Trans 44:1204-1208 (2003)

13. Corcoran, J., Nagy, P.B., Cawley, P.: Potential drop monitoring of creep damage at a weld. Rev. Prog. Quant. Nondestruct. Eval. 35. doi: $10.1063 / 1.4940625$

14. Jiles, D.C.: Introduction to Magnetism and Magnetic Materials, 2nd edn. CRC Press (1998)

15. Comsol Multiphysics, Comsol Inc. (2015)

16. Voss RF. 1/f (Flicker) Noise: A Brief Review. 33rd Annu Symp Freq Control 1979:40-6. doi:10.1109/FREQ.1979.200297

17. McMaster, R.C., McIntire, P., Mester, M.L. (eds.): Nondestructive Testing Handbook, vol. 4: Electromagnetic Testing, 2nd edn. McGraw-Hill Education (1986)

18. Model SR560, Low-Noise Preamplifier Manual (2013)

19. Smith, J.O.: Introduction to Digital Filters: With Audio Applications. W3K Publishing (2008)

20. Giacoletto, L.J.: Frequency- and time-domain analysis of skin effects. Magn. IEEE Trans. 32, 220-229 (1996)

21. Bowler, N.: Theory of four-point alternating current potential drop measurements on a metal half-space. J. Phys. D. Appl. Phys. 39, 584-589 (2006). doi:10.1088/0022-3727/39/3/024

22. Madhi, E., Nagy, P.B.: Sensitivity analysis of a directional potential drop sensor for creep monitoring. NDT\&E Int 44, 708-17 (2011). doi:10.1016/j.ndteint.2011.08.001

23. Sposito, G., Cawley, P., Nagy, P.B.: An approximate model for three-dimensional alternating current potential drop analyses using a commercial finite element code. NDT\&E Int 43, 134-40 (2010). doi:10.1016/j.ndteint.2009.10.002

24. Madhi, E.: In-situ creep monitoring using directional potential drop sensors. PhD Thesis, University of Cincinnati (2010)

25. Bowler, N., Bowler, J.R.: Theory of four-point alternating current potential drop measurements on conductive plates. Proc R Soc A 463, 817-36 (2007). doi:10.1098/rspa.2006.1791

26. Whitaker, J.C.: The Electronics Handbook. CRC Press (1996) 